(c) 2010 IEEE. Personal use of this material is permitted. Permission from IEEE must be obtained for all other uses, in any current or future media, including reprinting/republishing this material for advertising or promotional purposes, creating new collective works, for resale or redistribution to servers or lists, or reuse of any copyrighted component of this work in other works. 


\title{
Design of Amplify and Forward MIMO Relay Networks with QoS Constraint
}

\author{
Jafar Mohammadi†, Feifei Gaoł, and Yue Rong $\ddagger$ \\ ${ }^{\dagger}$ School of Engineering and Sciences, Jacobs University, Bremen, Germany \\ Email: $\{$ j.mohammadi, f.gao $\} @$ jacobs-university.de, \\ ${ }_{\ddagger}^{\ddagger}$ Department of Electrical and Computer Engineering, Curtin University of Technology, Australia \\ Email: y.rong@curtin.edu.au
}

\begin{abstract}
In this paper, we design the optimal precoding matrices for amplify-and-forward (AF) multiple-input multipleoutput (MIMO) relay networks. Specifically, we consider a dualhop relay network and minimize the total power consumed by source and relay under predetermined quality of service $(\mathrm{QoS})$ constraints, i.e., mean square error (MSE) constraints. By using majorization theory, we simplify the matrix-valued problem into a scalar-valued one. Since the problem is non-convex, we then propose two convex suboptimal problems that provide the upper and lower bound of the original objectives. Numerical results demonstrate that the lower bound and the upper bound are tight in high signal-to-noise ratio (SNR).
\end{abstract}

\section{INTRODUCTION}

Relay networks recently have drawn enormous research attentions because of their promising capability in achieving reliable communication and wide-ranging coverage for the next generation of wireless communication [1], [2]. Different types of relays from viewpoint of functionality were introduced. Regenerative relay, for example, detects the received data from transmitter before it forwards the data to the destination and is also called as decode-and-forward (DF) relay. Non-regenerative relay, on the other hand, only amplifies and forwards the received signal to the destination, and is named as amplify-and-forward (AF) relay. Non-regenerative relays have less computational complexity in comparison to the regenerative counterpart.

Considering the fact that multi-input multi-output (MIMO) technique [4] enhances the data transmission rate, combining relays and multiple antennas could further advance the future wireless communication systems. Most existing works in the area of designing MIMO relays focus on the AF relay networks, where a certain performance criterion is optimized subject to given power constraints at both the source and the relay. For example, the mutual information and the mean square error (MSE) criterions are selected as objective functions in [5], and [6], respectively. Applying the majorization theory [7], the authors of [8] recently proposed a unified frame work solution for most performance criteria. Nevertheless, the dual problem that is to minimize the consumed power subject to predetermined quality of service (QoS) constraints

The work is supported by the German Research Foundation (DFG) under Grant GA 1654/1-1.

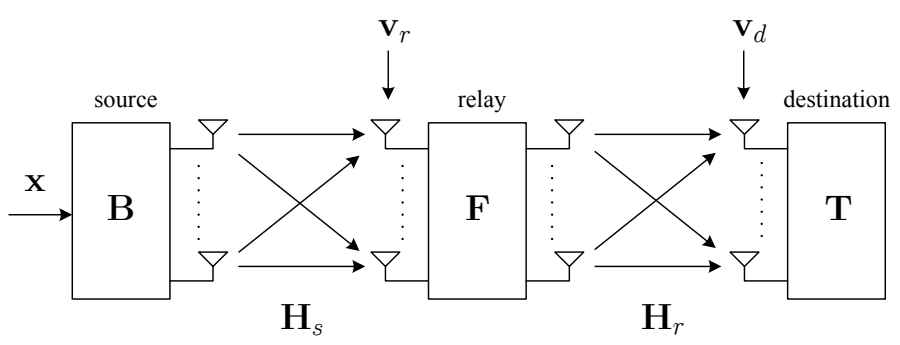

Fig. 1. A two-hop MIMO AF relay network.

has not been considered yet. Note that, QoS constraints play a prominent rule for a practical system design. For example in point-to-point MIMO case, [9] optimized the transmission power subject to a predefined set of QoS, i.e., MSE, signalto-noise ratio (SNR), and bit error rate (BER).

In this paper, we consider the two-hop MIMO AF relay networks and derive the optimal structure of both source precoding matrix as well as the relay amplifying matrix such that the overall transmission power from source and relay is minimized subject to a given set of QoS constraints. The extension to multi-hop MIMO AF relay networks can be found in [10]. Applying the majorization theory, the original matrix-valued non-convex problem can be reduced to a scalarvariable problem. The non-convexity of the problem prevent the application of the efficient optimization tools. Therefore, we refer to two convex suboptimal problems that serve as the upper bound and the lower bound of the original problem. Numerical results demonstrate that the lower bound and the upper bound are tight in high SNR range.

\section{PROBlem Formulation}

Consider a two-hop MIMO AF relay network shown in Fig. 1, where the transmitter, relay, and the receiver all have $L$ co-located antennas that cannot transmit and receive simultaneously. ${ }^{1}$ We assume that there is no direct link between the source and the destination. Furthermore, the flat fading channels are considered in our work. We denote the MIMO

\footnotetext{
${ }^{1}$ To simplify the discussion, we assume the same antennas in this paper. The extension to more general case can be found in journal version of this work.
} 
channels between source and relay by $\mathbf{H}_{s}$, while that between the relay and destination by $\mathbf{H}_{r}$. In this paper, we assume that the channels are perfectly known to all the nodes. Channel estimation for relay network has been discussed in [11].

Denote data streams transmitted from the source by the $L \times 1$ vector $\mathbf{x}$. In order to achieve the optimal design, we apply the precoding matrix $\mathbf{B}$ and $\mathbf{F}$ at the source and the relay, respectively. The signal received at the destination is

$$
\mathbf{z}=\mathbf{H}_{r} \mathbf{F} \mathbf{H}_{s} \mathbf{B} \mathbf{x}+\mathbf{H}_{r} \mathbf{F} \mathbf{v}_{r}+\mathbf{v}_{d},
$$

where $\mathbf{v}_{r}$ and $\mathbf{v}_{d}$ are additive white complex Gaussian noise at the relay and the destination, respectively, i.e., $\mathbf{v}_{r} \in$ $\mathcal{C N}\left(0, \sigma_{r}^{2} I_{N}\right)$ and $\mathbf{v}_{d} \in \mathcal{C N}\left(0, \sigma_{d}^{2} I_{N}\right)$. The data streams from the source are also considered to be independent from each other, i.e., $\mathrm{E}\left\{\mathbf{x x}^{H}\right\}=\mathbf{I}$. For simplicity in calculation, we set $\sigma_{d}=\sigma_{r}=1$.

Applying the linear minimum mean-square-error (MMSE) receiver at the destination whose corresponding decoding matrix is $\mathbf{T}$, the estimated data streams is denoted by

$$
\hat{\mathbf{x}}=\operatorname{Tr}
$$

For given $\mathbf{B}$ and $\mathbf{F}$, the optimal $\mathbf{T}$ is immediately obtained as [12]

$$
\begin{array}{r}
\mathbf{T}=\left(\mathbf{H}_{r} \mathbf{F} \mathbf{H}_{s} \mathbf{B}\left(\mathbf{H}_{r} \mathbf{F} \mathbf{H}_{s} \mathbf{B}\right)^{H}+\mathbf{H}_{r} \mathbf{F}\left(\mathbf{H}_{r} \mathbf{F}\right)^{H}+\mathbf{I}\right)^{-1} \\
\times \mathbf{H}_{r} \mathbf{F} \mathbf{H}_{s} \mathbf{B},
\end{array}
$$

while the corresponding error covariance is given by

$$
\begin{array}{r}
\mathbf{E}=\left[\mathbf{I}+\left(\mathbf{H}_{r} \mathbf{F} \mathbf{H}_{s} \mathbf{B}\right)^{H}\left(\mathbf{H}_{r} \mathbf{F}\left(\mathbf{H}_{r} \mathbf{F}\right)^{H}+\mathbf{I}\right)^{-1}\right. \\
\left.\times \mathbf{H}_{r} \mathbf{F} \mathbf{H}_{s} \mathbf{B}\right]^{-1} .
\end{array}
$$

In this paper, we formulate our QoS constraints as the MSE of each individual data streams. Suppose the $i$ th data stream should have MSE smaller than $\rho_{i}$ and define the QoS vector as $\boldsymbol{\rho}=\left[\rho_{1}, \ldots, \rho_{L}\right]^{T}$. Note that $\rho_{i}<1$ is required to avoid the trivial discussion. The QoS constraints can be written as

$$
\mathbf{E}_{i i} \leq \rho_{i}, \quad \text { or, } \quad \mathbf{d}\{\mathbf{E}\} \leq \boldsymbol{\rho},
$$

where $\mathbf{d}$ is the diagonal vector of $\mathbf{E}$ and the last " $\leq$ " denotes the element-wise operation.

On the other side, we define the objective function as the total power spent by the source and the relay. The power of the source is given by

$$
\mathrm{E}\left\{\operatorname{tr}\left(\mathbf{B} \mathbf{x x}{ }^{H} \mathbf{B}^{H}\right)\right\}=\operatorname{tr}\left(\mathbf{B B}^{H}\right),
$$

while the relay power is computed as

$$
\begin{aligned}
& \mathrm{E}\left\{\operatorname{tr}\left(\left(\mathbf{F} \mathbf{H}_{s} \mathbf{B} \mathbf{x}+\mathbf{F} \mathbf{v}_{r}\right)\left(\mathbf{F} \mathbf{H}_{s} \mathbf{B} \mathbf{x}+\mathbf{F} \mathbf{v}_{r}\right)^{H}\right)\right\} \\
= & \operatorname{tr}\left(\mathbf{F}\left(\mathbf{H}_{s} \mathbf{B} \mathbf{B}^{H} \mathbf{H}_{s}+\mathbf{I}\right) \mathbf{F}^{H}\right) .
\end{aligned}
$$

\section{Optimization With QOS CONSTANTS}

Before we start the section, let us define $\boldsymbol{\lambda}(\mathbf{X})=$ vector containing eigenvalues of $\mathbf{X}$. Not to mention that, one could go through the brief introduction on Majorization theory in Appendix first.

Based on the previous discussion, the optimal design is to find the best $\mathbf{B}$ and $\mathbf{F}$, such that the overall power consumption is minimized while the QoS constraints are satisfied.

Define

$$
\begin{aligned}
& \mathbf{M}=\mathbf{H}_{r} \mathbf{F H}_{s} \mathbf{B} \\
& \mathbf{R}=\left(\left(\mathbf{H}_{r} \mathbf{F}\right)\left(\mathbf{H}_{r} \mathbf{F}\right)^{\mathbf{H}}+\mathbf{I}\right)^{-1}
\end{aligned}
$$

The optimization can be described as

$$
\begin{aligned}
(\mathbf{P} 1): \quad \min _{\mathbf{B}, \mathbf{F}} & \operatorname{tr}\left(\mathbf{B B}^{H}\right)+\operatorname{tr}\left(\mathbf{F}\left(\mathbf{H}_{s} \mathbf{B B}^{H} \mathbf{H}_{s}+\mathbf{I}\right) \mathbf{F}^{H}\right) \\
\text { s.t. } & \mathbf{d}\left(\left(\mathbf{I}+\mathbf{M}^{H} \mathbf{R} \mathbf{M}\right)^{-1}\right) \leq \boldsymbol{\rho} .
\end{aligned}
$$

\section{A. Problem Conversion}

Let us define a new optimization first.

$$
\begin{aligned}
(\mathbf{P} 2): \min _{\tilde{\mathbf{B}}, \mathbf{F}} & \operatorname{tr}\left(\tilde{\mathbf{B}} \tilde{\mathbf{B}}^{H}\right)+\operatorname{tr}\left(\mathbf{F}\left(\mathbf{H}_{s} \tilde{\mathbf{B}} \tilde{\mathbf{B}}^{H} \mathbf{H}_{s}+\mathbf{I}\right) \mathbf{F}^{H}\right) \\
\text { s.t. } \quad & \mathbf{d}\left(\left(\mathbf{I}+\tilde{\mathbf{M}}^{H} \mathbf{R} \tilde{\mathbf{M}}\right)^{-1}\right) \succ^{w} \boldsymbol{\rho} \\
& \tilde{\mathbf{M}}=\mathbf{H}_{r} \mathbf{F} \mathbf{H}_{s} \tilde{\mathbf{B}} \\
& \tilde{\mathbf{M}}{ }^{H} \mathbf{R} \tilde{\mathbf{M}} \text { is diagonal }
\end{aligned}
$$

Theorem 1: Problem (P1) and (P2) have the same optimal value.

Proof:

Let $\mathbf{Z} \triangleq\left[\mathbf{I}+\mathbf{M}^{H} \mathbf{R M}\right]^{-1}$ and assume $\mathbf{Q}$ is the matrix of eigenvectors of $\mathbf{M}^{H} \mathbf{R M}$. We define $\tilde{\mathbf{B}}=\mathbf{B Q}$. Therefore, $\tilde{\mathbf{M}}^{H} \mathbf{R} \tilde{\mathbf{M}}$ is a diagonal matrix. The objective value in (P1) with $(\mathbf{B}, \mathbf{F})$ is the same as the objective value in (P2) with $(\tilde{\mathbf{B}}, \mathbf{F})$. Since $\mathbf{I}+\tilde{\mathbf{M}}^{H} \mathbf{R} \tilde{\mathbf{M}}$ is diagonal matrix, there is

$$
\begin{aligned}
\mathbf{d}\left(\left(\mathbf{I}+\tilde{\mathbf{M}}^{H} \mathbf{R} \tilde{\mathbf{M}}\right)^{-1}\right) & =\boldsymbol{\lambda}\left(\left(\mathbf{I}+\tilde{\mathbf{M}}^{H} \mathbf{R} \tilde{\mathbf{M}}\right)^{-1}\right) \\
=\boldsymbol{\lambda}\left(\left(\mathbf{I}+\mathbf{M}^{H} \mathbf{R M}\right)^{-1}\right) & =\boldsymbol{\lambda}(\mathbf{Z}) .
\end{aligned}
$$

Meanwhile, from $\mathbf{Z}_{i i} \leq \rho_{i}$ one can conclude $\mathbf{d}(\mathbf{Z}) \succ^{w} \boldsymbol{\rho}$. From Lemma 1 in Appendix, we know $\boldsymbol{\lambda}(\mathbf{Z}) \succ^{w} \mathbf{d}(\mathbf{Z})$. So there is $\boldsymbol{\lambda}(\mathbf{Z}) \succ^{w} \boldsymbol{\rho}$. Combing (12), we infer that for any feasible point $(\mathbf{B}, \mathbf{F})$ in $(\mathrm{P} 1)$, we can always find a corresponding feasible point $(\tilde{\mathbf{B}}, \mathbf{F})$ in $(\mathrm{P} 2)$ that gives the same objective value.

Next we prove the reverse part. Define $\tilde{\mathbf{Z}}=(\mathbf{I}+$ $\left.\tilde{\mathbf{M}}^{H} \mathbf{R} \tilde{\mathbf{M}}\right)^{-1}$. Assume that $(\tilde{\mathbf{B}}, \mathbf{F})$ is a feasible point of $(\mathrm{P} 2)$. Then we know that $\mathbf{d}(\tilde{\mathbf{Z}})=\boldsymbol{\lambda}(\tilde{\mathbf{Z}}) \succ^{w} \boldsymbol{\rho}$. From Lemma 2 in Appendix, we know that there exists a vector $\mathbf{c}$ such that both $\boldsymbol{\lambda}(\tilde{\mathbf{Z}}) \succ \mathbf{c}$ and $\mathbf{c} \leq \boldsymbol{\rho}$. From Lemma 1 we know that for each $\mathbf{c} \prec \boldsymbol{\lambda}(\tilde{\mathbf{Z}})$ there exists a matrix $\mathbf{W}$ with $\mathbf{d}(\mathbf{W})=\mathbf{c}$ and $\boldsymbol{\lambda}(\mathbf{W})=\boldsymbol{\lambda}(\tilde{\mathbf{Z}})$. Let $\mathbf{W}=\mathbf{Q}^{H} \tilde{\mathbf{Z}} \mathbf{Q}$ and define $\mathbf{B}=\tilde{\mathbf{B}} \mathbf{Q}$. Then $\mathbf{d}\left(\left(\mathbf{I}+\mathbf{M}^{H} \mathbf{R} \mathbf{M}\right)^{-1}\right)=\mathbf{d}(\mathbf{W}) \leq \boldsymbol{\rho}$. Moreover, the objective function of $(\mathrm{P} 1)$ with $(\mathbf{B}, \mathbf{F})$ is the same as the objective function of (P2).

Therefore, (P1) and (P2) always have the same optimal values. 
In following, we focus on solving the equivalent problem (P2) as the optimal values. The optimal precoding matrix $\mathbf{B}$ can be derived from $\tilde{\mathbf{B}}$ accordingly.

\section{B. Optimal Structure}

Since the CSI is known at all nodes, we can apply singular value decomposition (SVD) for different matrices. Throughout the paper, the singular values are assumed to be arranged in increasing order unless otherwise mentioned.

Let

$$
\begin{aligned}
& \mathbf{H}_{s}=\mathbf{U}_{s} \boldsymbol{\Lambda}_{s} \mathbf{V}_{s}^{H} \\
& \mathbf{H}_{r}=\mathbf{U}_{r} \boldsymbol{\Lambda}_{r} \mathbf{V}_{r}^{H}
\end{aligned}
$$

be the SVD of $\mathbf{H}_{s}$ and $\mathbf{H}_{r}$ respectively.

Suppose the ranks of $\mathbf{H}_{s}$ and $\mathbf{H}_{r}$ are $r_{s}$ and $r_{r}$, respectively. We can represent $\boldsymbol{\Lambda}_{s}$ and $\boldsymbol{\Lambda}_{r}$ as

$$
\begin{aligned}
\boldsymbol{\Lambda}_{s} & =\left[\begin{array}{cc}
\boldsymbol{\Sigma}_{s} & \mathbf{0}_{r_{s} \times\left(L-r_{s}\right)} \\
\mathbf{0}_{\left(L-r_{s}\right) \times r_{s}} & \mathbf{0}_{\left(L-r_{s}\right) \times\left(L-r_{s}\right)}
\end{array}\right], \\
\boldsymbol{\Lambda}_{r} & =\left[\begin{array}{cc}
\boldsymbol{\Sigma}_{r} & \mathbf{0}_{r_{r} \times\left(L-r_{r}\right)} \\
\mathbf{0}_{\left(L-r_{r}\right) \times r_{r}} & \mathbf{0}_{\left(L-r_{r}\right) \times\left(L-r_{r}\right)}
\end{array}\right],
\end{aligned}
$$

where $\boldsymbol{\Sigma}_{s}$ and $\boldsymbol{\Sigma}_{r}$ are square diagonal matrices containing all non-zero singular values of $\boldsymbol{\Lambda}_{s}$ and $\boldsymbol{\Lambda}_{r}$.

We further define the following two positive semi-definite (PSD) matrices for future use:

$$
\begin{aligned}
& \mathbf{X}=\mathbf{H}_{s} \tilde{\mathbf{B}} \tilde{\mathbf{B}}^{H} \mathbf{H}_{s}^{H}=\mathbf{U}_{X} \boldsymbol{\Lambda}_{X} \mathbf{U}_{X}^{H}, \\
& \mathbf{Y}=\mathbf{H}_{r} \mathbf{F}(\mathbf{X}+\mathbf{I}) \mathbf{F}^{H} \mathbf{H}_{r}^{H}=\mathbf{U}_{Y} \boldsymbol{\Lambda}_{Y} \mathbf{U}_{Y}^{H} .
\end{aligned}
$$

From (17) and (18), we can represent $\mathbf{H}_{s} \tilde{\mathbf{B}}$ and $\mathbf{H}_{r} \mathbf{F}$ as

$$
\begin{aligned}
& \mathbf{H}_{s} \tilde{\mathbf{B}}=\mathbf{U}_{X} \boldsymbol{\Lambda}_{X}^{\frac{1}{2}} \mathbf{V}_{X}^{H} \\
& \mathbf{H}_{r} \mathbf{F}=\mathbf{U}_{Y} \boldsymbol{\Lambda}_{Y}^{\frac{1}{2}} \mathbf{V}_{Y}^{H}(\mathbf{X}+\mathbf{I})^{-\frac{1}{2}},
\end{aligned}
$$

where $\mathbf{V}_{X}$ and $\mathbf{V}_{Y}$ can be any unitary matrices. However, it should be emphasized that $\mathbf{V}_{X}$ and $\mathbf{V}_{Y}$ are important parameters to the later optimizations.

From (19) and (20), we can represent $\tilde{\mathbf{B}}$ and $\mathbf{F}$ as

$$
\begin{aligned}
\tilde{\mathbf{B}} & =\mathbf{H}_{s}^{\dagger} \mathbf{U}_{X} \boldsymbol{\Lambda}_{X}^{\frac{1}{2}} \mathbf{V}_{X}^{H}=\mathbf{V}_{s} \boldsymbol{\Lambda}_{s}^{\dagger} \mathbf{U}_{s}^{H} \mathbf{U}_{X} \boldsymbol{\Lambda}_{X}^{\frac{1}{2}} \mathbf{V}_{X}^{H} \\
\mathbf{F} & =\mathbf{H}_{r}^{\dagger} \mathbf{U}_{Y} \boldsymbol{\Lambda}_{Y}^{\frac{1}{2}} \mathbf{V}_{Y}^{H}(\mathbf{X}+\mathbf{I})^{-\frac{1}{2}} \\
& =\mathbf{V}_{r} \boldsymbol{\Lambda}_{r}^{\dagger} \mathbf{U}_{r}^{H} \mathbf{U}_{Y} \boldsymbol{\Lambda}_{Y}^{\frac{1}{2}} \mathbf{V}_{Y}^{H}\left(\mathbf{U}_{X} \boldsymbol{\Lambda}_{X} \mathbf{U}_{X}^{H}+\mathbf{I}\right)^{-\frac{1}{2}},
\end{aligned}
$$

where $(\cdot)^{\dagger}$ denotes the pseudo-inverse of a matrix.

Therefore, instead of finding the optimal $\tilde{\mathbf{B}}$ and $\mathbf{F}$ directly, we will optimize according to the new variables $\mathbf{U}_{X}, \mathbf{V}_{X}$, $\mathbf{U}_{Y}, \mathbf{V}_{Y}, \Lambda_{Y}$ and $\Lambda_{Y}$.

1) Objective Function: After putting (21) and (22) back into the objective function in (P2), we obtain

$$
\begin{aligned}
\operatorname{tr}\left(\boldsymbol{\Lambda}_{s}^{\dagger} \mathbf{U}_{s}^{H} \mathbf{U}_{X} \boldsymbol{\Lambda}_{X} \mathbf{U}_{X}^{H} \mathbf{U}_{s} \boldsymbol{\Lambda}_{s}^{\dagger}\right) & \\
& +\operatorname{tr}\left(\boldsymbol{\Lambda}_{r}^{\dagger} \mathbf{U}_{r}^{H} \mathbf{U}_{Y} \boldsymbol{\Lambda}_{Y} \mathbf{U}_{Y}^{H} \mathbf{U}_{r} \boldsymbol{\Lambda}_{r}^{\dagger}\right) .
\end{aligned}
$$

Note that, the objective function is not depend on $\mathbf{V}_{X}$ and $\mathbf{V}_{Y}$, which gives us the freedom to find the optimal $\mathbf{V}_{X}$ and $\mathbf{V}_{Y}$ from the constraints only.
2) Constraints: Let us first look into the constraint structure. Applying matrix inversion lemma

$$
\begin{aligned}
& \left(\mathbf{A}_{1}-\mathbf{A}_{2} \mathbf{A}_{4}^{-1} \mathbf{A}_{3}\right)^{-1} \\
= & \mathbf{A}_{1}^{-1}+\mathbf{A}_{1}^{-1} \mathbf{A}_{2}\left(\mathbf{A}_{4}-\mathbf{A}_{3} \mathbf{A}_{1}^{-1} \mathbf{A}_{2}\right)^{-1} \mathbf{A}_{3} \mathbf{A}_{1}^{-1},
\end{aligned}
$$

we obtain

$$
\left(\mathbf{I}+\tilde{\mathbf{M}}^{H} \mathbf{R}^{-1} \tilde{\mathbf{M}}\right)^{-1}=\mathbf{I}-\underbrace{\tilde{\mathbf{M}}^{H}\left(\mathbf{R}^{-1}+\tilde{\mathbf{M}} \tilde{\mathbf{M}}^{H}\right)^{-1} \tilde{\mathbf{M}}}_{\mathbf{G}},
$$

where $\mathbf{G}$ is defined as the corresponding term.

Using the decomposition in (19) and (20), G can be rewritten as

$$
\begin{aligned}
\mathbf{G}=\mathbf{V}_{X} \boldsymbol{\Lambda}_{X}^{\frac{1}{2}}\left(\boldsymbol{\Lambda}_{X}+\right. & \mathbf{I})^{-\frac{1}{2}} \mathbf{U}_{X}^{H} \mathbf{V}_{Y} \boldsymbol{\Lambda}_{Y}^{\frac{1}{2}}\left[\boldsymbol{\Lambda}_{Y}+\mathbf{I}\right]^{-1} \\
& \times \boldsymbol{\Lambda}_{Y}^{\frac{1}{2}} \mathbf{V}_{Y}^{H} \mathbf{U}_{X}\left(\boldsymbol{\Lambda}_{X}+\mathbf{I}\right)^{-\frac{1}{2}} \boldsymbol{\Lambda}_{X}^{\frac{1}{2}} \mathbf{V}_{X}^{H} .
\end{aligned}
$$

From the diagonal constraint, we still need to properly choose $\mathbf{U}_{X}, \mathbf{V}_{X}$, and $\mathbf{V}_{Y}$ such that $\tilde{\mathbf{M}}^{H} \mathbf{R}^{-1} \tilde{\mathbf{M}}$ or equivalently $\mathbf{G}$ is diagonal. Thanks to the structure of $\mathbf{G}$ as well as the fact that $\mathbf{V}_{X}$ is not involved in the objective function, $\mathbf{U}_{X}$ and $\mathbf{V}_{Y}$ are not constrained by the diagonal requirement because for any $\mathbf{U}_{X}$ and $\mathbf{V}_{Y}$ we can always find a unitary matrix $\mathbf{V}_{X}$, such that $\mathbf{G}$ can be made into diagonal.

3) Optimal $\mathbf{V}_{X}$ and $\mathbf{V}_{Y}$ : Observing the constraint $\mathbf{d}(\mathbf{I}-$ $\mathbf{G}) \succ^{w} \boldsymbol{\rho}$, if we can find $\mathbf{V}_{Y}$ such that all of the components in $\mathbf{d}(\mathbf{I}-\mathbf{G})$ are minimized simultaneously, then this $\mathbf{V}_{Y}$ must be the optimal because it gives the largest possible freedom to optimize over the rest variables, i.e., $\mathbf{U}_{X}, \mathbf{U}_{Y}, \Lambda_{X}$, and $\Lambda_{Y}$. From [7, 9.H.2] on $\mathbf{G}$, we know

$$
\lambda[\mathbf{G}] \prec_{w} \mathbf{d}[\hat{\mathbf{G}}],
$$

where

$$
\hat{\mathbf{G}}=\boldsymbol{\Lambda}_{X}^{\frac{1}{2}}\left(\boldsymbol{\Lambda}_{X}+\mathbf{I}\right)^{-\frac{1}{2}} \boldsymbol{\Lambda}_{Y}^{\frac{1}{2}}\left[\boldsymbol{\Lambda}_{Y}+\mathbf{I}\right]^{-1} \boldsymbol{\Lambda}_{Y}^{\frac{1}{2}}\left(\boldsymbol{\Lambda}_{X}+\mathbf{I}\right)^{-\frac{1}{2}} \boldsymbol{\Lambda}_{X}^{\frac{1}{2}},
$$

is the multiplication of the diagonal matrices in (26). Moreover, (27) can also be written as

$$
\lambda[\mathbf{I}-\mathbf{G}] \prec^{w} \mathbf{d}[\mathbf{I}-\hat{\mathbf{G}}] .
$$

Since $\mathbf{V}_{X}$ is designed to make $\mathbf{G}$ diagonal and also from Lemma 1, we can rewrite (29) as

$$
\mathbf{d}[\mathbf{I}-\mathbf{G}] \prec^{w} \mathbf{d}[\mathbf{I}-\hat{\mathbf{G}}],
$$

so $\mathbf{d}[\mathbf{I}-\hat{\mathbf{G}}]$ simultaneously minimize all the component in $\mathbf{d}[\mathbf{I}-\mathbf{G}]$. Meanwhile, $\mathbf{G}=\hat{\mathbf{G}}$ if $\mathbf{V}_{Y}=\mathbf{U}_{X}$ and $\mathbf{V}_{\mathbf{X}}=\mathbf{I}$. Therefore, $\mathbf{V}_{Y}=\mathbf{U}_{X}$ and $\mathbf{V}_{\mathbf{X}}=\mathbf{I}$ must be the optimal solution to $\mathbf{V}_{Y}$.

4) Optimal $\mathbf{U}_{X}$ and $\mathbf{U}_{Y}$ : Interestingly, the unitary matrices $\mathbf{U}_{X}$ and $\mathbf{U}_{Y}$ in objective function are not related with the constraint. Therefore, one can find the optimal $\mathbf{U}_{X}$ and $\mathbf{U}_{Y}$ from unconstrained optimization

We will need the following matrix inequality [7] to proceed: Given two $L \times L$ positive semi-definite matrices $\mathbf{A}_{1}$ and 
$\mathbf{A}_{2}$ with eigenvalues $\lambda_{l}\left(\mathbf{A}_{1}\right)$ and $\lambda_{l}\left(\mathbf{A}_{2}\right)$ arranged in the increasing order, then

$$
\sum_{l=1}^{L} \lambda_{l}\left(\mathbf{A}_{1}\right) \lambda_{L-l+1}\left(\mathbf{A}_{2}\right) \leq \operatorname{tr}\left(\mathbf{A}_{1} \mathbf{A}_{2}\right) \leq \sum_{l=1}^{L} \lambda_{l}\left(\mathbf{A}_{1}\right) \lambda_{l}\left(\mathbf{A}_{2}\right)
$$

Based on LHS of (30), the first term in the objective function satisfies

$$
\operatorname{tr}\left(\boldsymbol{\Lambda}_{s}^{\dagger} \mathbf{U}_{s}^{H} \mathbf{U}_{X} \boldsymbol{\Lambda}_{X} \mathbf{U}_{X}^{H} \mathbf{U}_{s} \boldsymbol{\Lambda}_{s}^{\dagger}\right) \geq \operatorname{tr}\left(\boldsymbol{\Lambda}_{s}^{\dagger} \boldsymbol{\Lambda}_{X} \boldsymbol{\Lambda}_{s}^{\dagger}\right)
$$

and the minimum is achieved when $\mathbf{U}_{X}=\mathbf{U}_{s}$ because the diagonal elements in $\boldsymbol{\Lambda}_{s}$ and $\boldsymbol{\Lambda}_{X}$ are all arranged in increasing order.

Same discussion holds for the second term in the objective function, i.e.,

$$
\operatorname{tr}\left(\boldsymbol{\Lambda}_{r}^{\dagger} \mathbf{U}_{r}^{H} \mathbf{U}_{Y} \boldsymbol{\Lambda}_{Y} \mathbf{U}_{Y}^{H} \mathbf{U}_{r} \boldsymbol{\Lambda}_{r}^{\dagger}\right) \geq \operatorname{tr}\left(\boldsymbol{\Lambda}_{r}^{\dagger} \boldsymbol{\Lambda}_{Y} \boldsymbol{\Lambda}_{r}^{\dagger}\right)
$$

and the minimum is achieved when $\mathbf{U}_{Y}=\mathbf{U}_{r}$.

5) Optimal $\Lambda_{X}$ and $\Lambda_{Y}$ : Now we only need to optimize over $\boldsymbol{\Lambda}_{X}$ and $\boldsymbol{\Lambda}_{Y}$. Substituting the optimal $\mathbf{U}_{X}, \mathbf{U}_{Y}, \mathbf{V}_{X}$, and $\mathbf{V}_{Y}$ into the objective function and the constraints, we obtain

$$
\begin{aligned}
\min _{X}, \boldsymbol{\Lambda}_{Y} & \operatorname{tr}\left(\boldsymbol{\Lambda}_{s}^{\dagger} \boldsymbol{\Lambda}_{X} \boldsymbol{\Lambda}_{s}^{\dagger}\right)+\operatorname{tr}\left(\boldsymbol{\Lambda}_{r}^{\dagger} \boldsymbol{\Lambda}_{Y} \boldsymbol{\Lambda}_{r}^{\dagger}\right) \\
\text { s.t. } & \mathbf{I}-\boldsymbol{\Lambda}_{X} \boldsymbol{\Lambda}_{Y}\left(\boldsymbol{\Lambda}_{X}+\mathbf{I}\right)^{-1}\left(\boldsymbol{\Lambda}_{Y}+\mathbf{I}\right)^{-1} \succ^{w} \boldsymbol{\rho} .
\end{aligned}
$$

Since all of the matrices in (34) are diagonal, the optimization can be simplified to a scalar one.

Define $a_{l}$ and $b_{l}$ as the $l$ th diagonal entries of $\left(\boldsymbol{\Lambda}_{s}^{\dagger}\right)^{2}$ and $\left(\boldsymbol{\Lambda}_{r}^{\dagger}\right)^{2}$ respectively. Further define $x_{l}$ and $y_{l}$ as the $l$ th diagonal entries of $\boldsymbol{\Lambda}_{X}$ and $\boldsymbol{\Lambda}_{Y}$ respectively.

Then the optimization (34) is converted to

$$
\begin{aligned}
\min _{x_{l}, y_{l}} & \sum_{l=1}^{L} a_{l} x_{l}+b_{l} y_{l} \\
\text { s.t. } & \sum_{l=1}^{k} \frac{y_{l}+x_{l}+1}{y_{l}+x_{l}+y_{l} x_{l}+1} \leq \sum_{l=1}^{k} \rho_{l} \quad k=1, \ldots, L, \\
& x_{l} \geq 0, y_{l} \geq 0, \quad \forall l .
\end{aligned}
$$

Unfortunately, the constraint in problem (35) is not convex which makes the optimal solution hard to compute.

Therefore we propose two convex bounds on the constraints, i.e.,

$$
\begin{aligned}
& \frac{y_{i}+x_{i}+1}{y_{i}+x_{i}+y_{i} x_{i}+1} \leq \frac{y_{i}+x_{i}+2}{y_{i}+x_{i}+y_{i} x_{i}+1}, \\
& \frac{y_{i}+x_{i}+1}{y_{i}+x_{i}+y_{i} x_{i}+1} \geq \frac{y_{i}+x_{i}}{y_{i}+x_{i}+y_{i} x_{i}} .
\end{aligned}
$$

If we replace the constraint by its upperbound in (36), we obtain an upper bound in the objective value because we reduce the feasible region. On the other hand, If we replace the constraint by its lower bound in (37), we obtain a lower bound in the objective value.

Note that at high SNR, the upper bound (36) and the lower bound (37) approach each other. From the squeeze rule, we

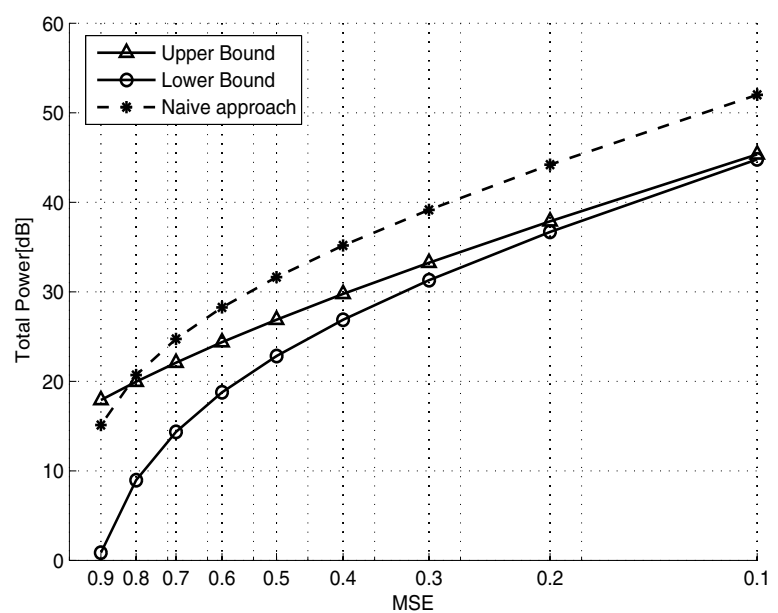

Fig. 2. Performance with equal QoS constraint

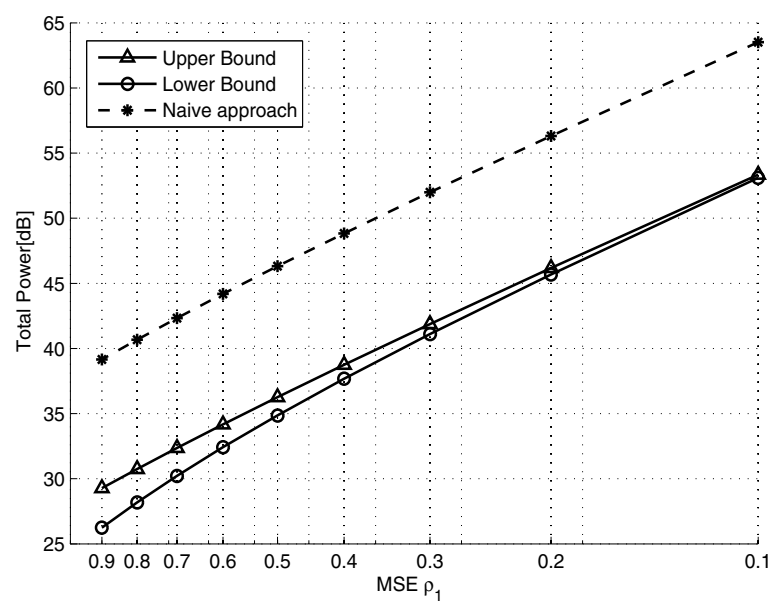

Fig. 3. Performance with unequal QoS constraint, $\rho_{1}=2 \rho_{2}=2 \rho_{3}=$ $2 \rho_{4}=4 \rho_{5}=4 \rho_{6}$,

know that the true objective value can be determined from a very small region.

The optimization with either (36) or (37) are convex and can be solved by efficient interior point method [13].

\section{Numerical Results}

In this section, we provide numerical results to corroborate our proposed studies. For all of the examples, we assume that the channel matrices $\left(\mathbf{H}_{r}\right.$ and $\left.\mathbf{H}_{s}\right)$ have independent and identically distributed Gaussian entries with zero-mean and variance 1 . We take the number of the antennas as 6 , and 1000 Monte-Carlo runs are taken for averaging the results. We used CVX convex optimization toolbox [14] for MATLAB, to solve these optimization problems.

In the first example, we consider identical MSE requirements for all the data steams by using the same $\rho_{l}$. We compare the optimal solutions from both the upper bound constraint and the lower bound constraint with a naive method where 
the power allocation $x_{l}$ and $y_{l}$ take the same value. The total power from different scheme versus $\rho_{l}$ is displayed in Fig. 2. We observe that for higher level of QoS, i.e, smaller MSE, the difference between the power lower-bound and power upperbound decreases. For example, at MSE $=0.2$, the difference is less one $\mathrm{dB}$. This is also consistent with our previous claim for the high SNR region. From the squeeze rule, the true objective value can be more easily guessed in high SNR. However, the naive method requires more than $6 \mathrm{~dB}$ power to achieve the same QoS requirement.

In the next example, we consider unequal levels of QoS for different data streams as $\rho_{1}=2 \rho_{2}=2 \rho_{3}=2 \rho_{4}=4 \rho_{5}=4 \rho_{6}$. Fig. 3 illustrates the total power verus $\rho_{1}$ for three different methods. It is observed that, the difference between the lower and upper bound is reduced in unequal QoS constraints, even in lower SNR region. So the true optimal values is better bounded as compared to the identical QoS case. Secondly, the difference to the naive method increase to as much as $10 \mathrm{~dB}$, which indicates the superior property of the proposed methods than the naive method, and imply the necessity of the proposed study.

\section{CONClusion}

In this paper, we considered optimal design of the precoding matrix in an MIMO AF relay network. The total source and relay power consumption is minimized subject to a predetermined set of QoS constraints. We first applied the majorization theory to simplify the matrix-valued optimization into a scalar one, and then propose to convex suboptimal problems that serve as the upper and lower bounds of our original problems. Simulations shows that the proposed bounds are tight at high SNR region or when the QoS for different data streams are unequal. Moreover, the proposed method performs much better than the naive power allocation.

\section{APPENDIX}

In this section we briefly introduce the basic notion of majorization theory. Comprehensive discussion can be found in [7]. let

Definition 1: If for any vector $\mathbf{x}=\left[x_{1}, x_{2}, \ldots, x_{n}\right] \in \mathbb{R}^{n}$,

$$
x_{(1)} \leq \ldots \leq x_{(n)}
$$

represents the elements of $\mathrm{x}$ in increasing order. Similarly, assume

$$
x_{[1]} \geq \ldots \geq x_{[n]}
$$

represents the elements of $\mathrm{x}$ in decreasing order.

Definition 2: For any $\mathbf{x}, \mathbf{y} \in \mathbb{R}^{n}, \mathbf{x}$ is majorized by $\mathbf{y}$ if

$$
\begin{aligned}
\sum_{i=1}^{k} x_{(i)} & \geq \sum_{i=1}^{k} y_{(i)}, \quad 1 \leq k \leq n-1 \\
\sum_{i=1}^{n} x_{(i)} & =\sum_{i=1}^{n} y_{(i)},
\end{aligned}
$$

and is denoted by $\mathbf{x} \prec \mathbf{y}$, or $\mathbf{y} \succ \mathbf{x}$.
Definition 3: [7] For any $\mathbf{x}, \mathbf{y} \in \mathbb{R}^{n}, \mathbf{x}$ is weakly supermajorized by $\mathbf{y}$ if

$$
\sum_{i=1}^{k} x_{(i)} \geq \sum_{i=1}^{k} y_{(i)}, \quad 1 \leq k \leq n
$$

We denote this with $\mathbf{x} \prec{ }^{w} \mathbf{y}$ (or equivalently $\mathbf{y} \succ^{w} \mathbf{x}$ ). Also, $\mathbf{x}$ is weakly submajorized by $\mathbf{y}$ if

$$
\sum_{i=1}^{k} x_{[i]} \leq \sum_{i=1}^{k} y_{[i]}, \quad 1 \leq k \leq n
$$

We denote this with $\mathbf{x} \prec_{w} \mathbf{y}$ (or equivalently $\mathbf{y} \succ_{w} \mathbf{x}$ ).

Lemma 1 ( [7],9.B.1): Let $\mathbf{M}$ be an $n \times n$ Hermitian matrix with a vector $\mathbf{d}(\mathbf{M})$ denoting its diagonal elements and let vector $\boldsymbol{\lambda}(\mathbf{M})$ contain its eigenvalues. Then

$$
\overline{\mathbf{d}}(\mathbf{M}) \prec \mathbf{d}(\mathbf{M}) \prec \boldsymbol{\lambda}(\mathbf{M})
$$

where $\overline{\mathbf{d}}(\mathbf{M})_{i}=\operatorname{mean}(\mathbf{d}(\mathbf{M}))$.

Reversely, given vectors $\mathbf{a}$ and $\mathbf{b}$ with $\mathbf{a} \prec \mathbf{b}$, then there exists an $n \times n$ Hermitian matrix $\mathbf{M}$ which its diagonal elements are $\mathbf{a}$ and its eigenvalues are $\mathbf{b}$.

Lemma 2 ( [7],5.A.9): For any $\mathbf{a}$ and $\mathbf{b}$ satisfying $\mathbf{a} \prec^{w} \mathbf{b}$, there exists a vector $\mathbf{x}$ such that

$$
\begin{gathered}
\mathbf{x} \prec \mathbf{b}, \quad \mathbf{a} \geq \mathbf{x} . \\
\text { REFERENCES }
\end{gathered}
$$

[1] J. N. Laneman and G. W. Wornell, "Distributed space time block coded protocols for exploiting cooperative diversity in wireless networks," IEEE Trans. Inform. Theory, vol. 49, pp. 2415-2425, Oct. 2003.

[2] J. N. Laneman, D. N. C. Tse, and G. W. Wornell, "Cooperative diversity in wireless networks: efficient protocols and outage behavior," IEEE Trans. Inform. Theory, vol. 50, pp. 3062-3080, Dec. 2004.

[3] A. S. Behbahani, R. Merched and A. M. Eltawil, "Optimization of a MIMO relay network," IEEE Trans. SignalProcessing, vol. 56, pp. 50625073, Oct. 2008.

[4] I. E. Telatar, "Capacity of multi-antenna Gaussian channels," Eur. Trans. Telecom., vol. 10, pp. 585-595, Nov. 1999.

[5] X. Tang and Y. Hua,"Optimal design of non-regenerative MIMO wireless relays," IEEE Trans. Wireless Commun., vol.6, no.4, pp.1398-1407, Apr. 2007.

[6] I. Hammerstrom and A. Wittneben,"Power allocation schemes for amplify-and-forward MIMO-OFDM relay links," IEEE Trans. Wireless Commun., vol.6, no.8, pp.2798-2802, Aug. 2007.

[7] A. W. Marshall and I. Olkin, Inequalities: Theory of Majorization and Its Applications. New York: Academic, 1979.

[8] Y. Rong, X. Tang, and Y. Hua,"A Unified framework for optimizing Linear Non-Regenerative Multicarrier MIMO Relay Communication Systems," IEEE Trans. Signal Processing, vol. 57, pp. 4837-4851, Dec. 2009.

[9] D. P. Palomar, Miguel Angel Lagunas, and John M. Cioffi, "Optimum linear joint transmit-receive processing for MIMO channels with QoS constraints," IEEE Trans. on Signal Processing, vol. 52, no. 5, pp. 11791197, May 2004.

[10] Y. Rong,"Multi-hop non-regenerative MIMO relays-QoS considerations," IEEE Trans. Commun., revised, Mar. 2009.

[11] F. Gao, T. Cui, and A. Nallanathan, "On channel estimation and optimal training design for amplify and forward relay network," IEEE Trans. Wireless Commun., vol. 7, no. 5, pp. 1907-1916, May 2008.

[12] S. M. Kay, Fundumentals of Statistical Signal Processing: Estimation Theory. Englewood Cliffs, NJ: Prentice-Hall, 1993.

[13] S. Boyd and L. Vandenberghe, Convex Optimization, Cambridge University Press, 2004.

[14] M. Grant and S. Boyd. CVX: Matlab software for disciplined convex programming (web page and software). http://stanford.edu/ boyd/cvx, June 2009. 